\title{
Questes
}

\section{Un texte entre orthodoxie et hérésie : le Mirouer des simples ames (XIII ${ }^{\mathrm{e}}$ siècle)}

\section{Elodie Pinel}

\section{OpenEdition}

\section{Journals}

Édition électronique

URL : http://journals.openedition.org/questes/4340

DOI : $10.4000 /$ questes. 4340

ISSN : 2109-9472

\section{Éditeur}

Les Amis de Questes

\section{Édition imprimée}

Date de publication : 6 mai 2016

Pagination : 69-83

ISSN : 2102-7188

\section{Référence électronique}

Elodie Pinel, « Un texte entre orthodoxie et hérésie:

le Mirouer des simples ames (XIII siècle) », Questes [En ligne], 32 | 2016, mis en ligne le 10 mai 2016,

consulté le 19 avril 2019. URL : http://journals.openedition.org/questes/4340 ; DOI : 10.4000/ questes. 4340

Ce document a été généré automatiquement le 19 avril 2019

(c) Association des amis de «Questes » 


\title{
Un texte entre orthodoxie et hérésie :
}

\section{le Mirouer des simples ames (XIII siècle)}

\author{
Elodie Pinel
}

Orthodoxie et hérésie sont des notions relatives. Au cœur de leur relation se trouve, dans l'Occident chrétien, la communauté de l'Église de Rome. Là où l'orthodoxie garantit le maintien de la communauté chrétienne, l'hérésie menace sa cohésion de deux manières : hétérodoxe, elle introduit le doute et entraîne le relâchement des liens de confiance qui maintiennent la communauté; insoumise, elle conteste l'organisation hiérarchique qui structure cette communauté. C'est ce second aspect qui prime à partir du XII siècle, où l'enjeu de l'Église n'est plus «la construction de la doctrine» mais «la direction des fidèles dans un monde politiquement divisé auquel la notion de chrétienté apporte une unité ${ }^{~}$. À cette « atteinte au bon ordre de la société chrétienne ${ }^{2}$ » doit répondre un rejet salutaire, nécessaire pour prévenir la contagion : l'excommunication qui place l'hérétique hors de la communauté.

2 Le Mirouer des simples ames est un texte de spiritualité du XIII siècle attribué à la béguine Marguerite Porete. Du Mirouer, que nous désignons ici par son titre médio-français par commodité, nous connaissons quatorze manuscrits. Ces manuscrits, datant tous des XIV et $\mathrm{XV}^{\mathrm{e}}$ siècles, portent un texte écrit en latin, en anglais médiéval, en italien médiéval ou en moyen français ${ }^{3}$. Cet écrit didactique et spirituel traite d'une communauté religieuse double : celle de l'Église et celle de l'« eslite ", Église dans l'Église ${ }^{4}$. Or le Mirouer des simples ames met en question le maintien de la communauté religieuse chrétienne : son auteur cherche à faire preuve d'allégeance à l'Église et d'orthodoxie mais son texte semble critiquer le clergé et peut être soupçonné d'hétérodoxie. Cette tension, nous allons l'examiner dans l'ensemble du texte puis la voir à l'œuvre dans le chapitre, particulièrement significatif, consacré à l'Eucharistie, avant d'élargir notre examen à sa persistance lors de la réception du texte $\mathrm{du} \mathrm{XIV}^{\mathrm{e}} \mathrm{au} \mathrm{XVI}^{\mathrm{e}}$ siècle. Ces deux premiers points 
nous feront adopter la perspective d'un membre de la communauté religieuse chrétienne et le dernier celle des garants de cette communauté.

\section{Un texte entre maintien et rejet de l'autorité du clergé}

3 Le Mirouer recèle bien des points difficiles, surtout sur le plan doctrinal. Bien qu'il affirme souvent s'inscrire dans la communauté chrétienne, ce texte se démarque parfois par une attitude plus qu'ambiguë à l'égard des rites et hiérarchies qui la cimentent. L'Église cherche à assurer, pour le maintien de sa communauté, le respect des dogmes et des sacrements, d'une part, et l'obéissance à l'autorité de son clergé, d'autre part. Or le Mirouer adopte parfois une position ambivalente à l'égard de l'obéissance due aux garants de cette communauté, alors même que son auteur semble par ailleurs prêter allégeance à l'Église.

Cette volonté d'obéir au clergé s'affiche ainsi clairement dans un passage précis du Mirouer. L'auteur y indique avoir recherché l'approbation de plusieurs hommes d'Église. Nous reproduisons notre traduction qui tente de concilier les différentes leçons des traditions manuscrites italienne, anglaise, latine et médio-française :

Moi, créature créée par celui qui crée, moyen par lequel le Créateur fit de lui-même ce livre, pour qui je ne sais ni ne veux [le] savoir, parce que ceci je ne dois pas [le] vouloir. Il me suffit en effet de savoir que cela est dans le secret de la divine sagesse et dans l'espérance. Je les salue toutefois, par amour de la paix de charité dans la très haute Trinité, qui daigne les diriger, en leur disant le témoignage de [sa ?] vie comme l'attestent (per recordationem) les clercs qui entendirent (audierunt) ce livre ${ }^{5}$.

Dans ce passage, l'auteur salue le groupe des destinataires de son livre, les « simples ames ${ }^{6}$ ", qu'elle ne connaît pas, et évoque les garants du maintien de la communauté chrétienne : les «clericorum qui audierunt istum librum ${ }^{7} »$. Les noms de ces clercs et les discours d'approbation tenus au sujet du livre sont ensuite indiqués. Le chapitre se clôt en expliquant ce pour quoi de telles approbations avaient été recherchées : elles visaient à garantir la "paix» des auditeurs. Écrites, ces attestations gardent le même but, concernant, cette fois, les destinataires du texte, ses lecteurs futurs. L'histoire de ce texte semble donc se diviser en deux époques : la première, celle d'une diffusion orale, donnant lieu à des suspicions d'hérésie contre laquelle l'auteur voulut se protéger et protéger ses auditeurs et la seconde, celle d'une diffusion écrite, anticipant de futures persécutions et cherchant à en prémunir les lecteurs. Nous sommes donc en présence d'un texte se voulant soumis à l'Église, certes, mais ayant été suspecté d'hérésie, avant tout.

7 Mise à part cette recherche d'approbation, que dit le texte de l'Église et du clergé ? Est-il critique à l'égard de ces clercs sollicités pour garantir la "paix» des auditeurs et lecteurs? Paradoxalement, la réponse à cette question semble affirmative. Elle apparaît dès le poème liminaires:

Theologiens ni aultres clers,

Point n'en aurez l'entendement

Tant aiez les engins clers

Se n'y procedez humblement

[...] Humiliez dont voz sciences

Qui sont de Raison fondees,

Et mettez toutes vos fiances

En celles qui sont donnees 
D’Amour, par Foy enluminees,

Et ainsy comprendrez ce livre

Qui d'Amour fait l'Ame vivre.

8 L'apostrophe aux théologiens et aux clercs est directe, l'avertissement est limpide : ce n'est pas par la raison, outil principal des clercs, que l'on comprend l'amour de Dieu, mais par l'humilité et la foi.

9 Ce poème liminaire n'apparaît cependant que dans le manuscrit de Chantilly. Il s'agit donc vraisemblablement d'une pièce rajoutée à l'ensemble, qui entend refléter une intention de l'auteur alors qu'elle n'est pas aussi claire dans le texte. En effet, seul le chapitre 121 explicite une critique des clercs : « Il n'y a si grant clerc ou monde / Qui vous en sceust parler [de la noblesse qu'il y a à être anéanti] ${ }^{9}$ ». En revanche, si le personnage allégorique de Raison est très présent et joue le mauvais rôle, il n'est pas ouvertement identifié avec les clercs et théologiens. Raison et Amour semblent s'opposer comme deux puissances internes à toute âme plutôt que comme deux entités réelles. En somme, dans le Mirouer, qui dit critique de la raison ne dit pas explicitement critique du clergé : cette dernière existe, mais elle reste discrète.

Le Mirouer est donc un texte ambigu : souhaitant certes constituer au sein de l'Église une communauté d'élus, l'auteur ne rejette pas pour autant la communauté chrétienne. Elle cherche bien plutôt à s'y inclure et à ne pas faire courir à ses auditeurs et lecteurs le risque d'en être exclus par une potentielle accusation d'hétérodoxie. Mais, ce faisant, elle ne renonce pas à son souhait de suivre une voie d'excellence spirituelle qu'on peut qualifier d'élitiste, et partant de séditieuse, parce que recherchant une union exclusive avec Dieu en refusant l'autorité de l'Église comme intermédiaire nécessaire ${ }^{10}$. N'est-ce pas la définition même de l'hérésie ? Les hérétiques «n'acceptaient pas que la piété fût une institution, qu'il faille pour communiquer avec le divin l'entremise des prêtres ${ }^{11}$ ». Les franciscains Spirituels, puis les lollards et les hussites à la suite de John Wyclif, opposeront à l'Église de Rome, l'Église invisible des élus. Le Mirouer, en définissant l'Église autrement que comme une communauté de fidèles subordonnée à un petit groupe de clercs, partage ces positions et peut à ce titre être soupçonné l'hérésie.

\section{Communion et maintien de la communauté}

11 On trouve dans le Mirouer des développements théologiques qui semblent à première vue tout à fait conformes à l'orthodoxie de son temps. Mais le sont-ils vraiment ? Pour traiter cette question, nous allons examiner le rapport du Mirouer à l'acte qui symbolise, rappelle et renforce la cohésion de la communauté chrétienne : la communion. Rappelons que sa définition a été fixée par le concile de Latran IV en $1215^{12}$. Notre texte y consacre le chapitre 15. Nous nous situons alors au tout début de l'œuvre ${ }^{13}$. L'enjeu de la discussion théologique sur le Saint Sacrement revêt une importance particulière au XIII $^{\mathrm{e}}$ siècle, moment où la dévotion des fidèles envers ce sacrement se développe fortement, surtout parmi les laïcs ${ }^{14}$. Les procès en hérésie de l'époque reprochent notamment aux accusés de se soustraire à la communion ou de ne pas regarder l'hostie et le calice portés par le prêtre pendant leur consécration lors de l'office. La profanation de l'hostie est même considérée par l'Église comme un déicide ${ }^{15}$. Or c'est par la communion que les fidèles manifestent leur soumission à l'autorité spirituelle du clergé et leur appartenance à la communauté chrétienne. Dès lors, en proposer une interprétation divergente revient à mettre en péril le maintien de la communauté. 
12 Le chapitre du Mirouer traitant de l'Eucharistie présente le dialogue de plusieurs personnages allégoriques : le personnage «Lumière de Foy» entend expliquer le Saint Sacrement au moyen d'une comparaison, méthode pédagogique utilisée notamment à l'université. L'exposé commence par une question étrange : Foy demande si le Christ est toujours dans le « sacrement » (c'est-à-dire dans l'hostie) lorsque celle-ci est mise dans un mortier et broyée, hypothèse soulevée par «Lumiere de Foy ». « Foy » et « Temptacion » jouent le rôle des interlocuteurs et Foy oppose, en conclusion, la vision de la foi à celle des sens : le Christ n'est pas vu dans le pain par les sens mais par la foi. Sa présence n'en est pas moins « réelle» mais ce terme, qui est au cœur du canon de Latran IV, n'est pas utilisé : le chapitre 15 ne reprend pas la lettre du concile ${ }^{16}$.

Revenons sur cette étrange hypothèse soulevée par «Lumière de Foy »: "Prenez ce Sacrement, mectez le en ung mortier avec aultres choses, et breez ce Sacrement tant que vous n'y puissez point veoir ne point sentir de la Personne que vous y avez $\mathrm{mis}^{17}$ ". Ce à quoi répond Foy : "Je vous dy vrayement que il n'y est mie ${ }^{18}$ ». Cette hypothèse, étrange en ce qu'elle évoque une opération sacrilège à l'égard de l'hostie, dérange l'orthodoxie. S'agirait-il d'une question quodlibétique? Plusieurs questions quodlibétiques recensées dans la base Quodlibase abordent la question de l'eucharistie ${ }^{19}$; parmi elles, seul le Quodlibet IV, 23 de Pierre de Jean Olivi examine le cas d'une hostie broyée, utilisée en l'occurrence comme engrais par une femme, le théologien se demandant si le Christ est encore présent dans les miettes du pain transsubstantié ${ }^{20}$. La possibilité d'une leçon théologique ayant pour source la référence des Franciscains spirituels est intéressante : elle tendrait à confirmer la connaissance par l'auteur du Miroir de ce courant de pensée et de spiritualité. D'autres hypothèses peuvent être soulevées : le Mirouer peut se faire l'écho du problème de la manducation de l'hostie ou encore de sa destruction lors du miracle de Billettes selon lequel, en 1270, un juif, voulant éprouver le sacrement eucharistique, aurait poignardé une hostie et l'aurait plongée dans l'eau bouillante ${ }^{21}$. Il l'aurait alors vue devenir écarlate du sang du Christ. Ce miracle, prouvant la présence réelle du Christ dans l'hostie, aurait été à l'origine de sa conversion. Signalons que de nombreux prédicateurs examinaient une "question théologale » devant leur auditoire et que le cas du mauvais usage d'une hostie pouvait être abordé lors d'un sermon ${ }^{22}$. Dans ce chapitre, le Mirouer, sans contester ouvertement un des points les plus cruciaux de ce que l'Église définit comme orthodoxe, relate des pratiques qui détournent l'Eucharistie de son symbole de communion des fidèles et pouvait être lu comme tendant vers l'hérésie.

Si l'on s'en tient à la lettre du texte, déterminer s'il a réellement menacé la communauté chrétienne, dont l'Église est garante, n'est pas simple ; cela ne l'est pas non plus si l'on en observe les copies. En effet, seuls les manuscrits en médio-anglais et en médio-français portent les paragraphes précédemment cités du chapitre 15. On ne trouve pas traces de ces passages dans les versions latines et italiennes. Quelle conclusion en tirer? L'hypothèse a minima est celle d'un manuscrit-source ayant sauté involontairement ces paragraphes, mais il s'agirait là d'un oubli bien conséquent et bien significatif. Aussi estce l'hypothèse d'un retranchement de ce passage des versions latines, dont dérivent les versions italiennes, qui s'impose : ce passage aurait été perçu comme problématique par un certain lectorat, qui aurait préféré nettoyer le texte pour en permettre la copie à côté de grands noms de la mystique. Cette hypothèse soulève pourtant un problème: comment expliquer que les versions médio-anglaises et médio-française conservent ce passage? Ne semblait-il pas problématique à ces copistes et lecteurs ? À examiner les manuscrits, on observe que le chapitre est signalé en marge par l'indication « Sur le Saint 
Sacrement» ou "Sur le Sacrement de l'autel », ce qui indique une lecture tabulaire. Aucune note marginale ne vient contester ou souligner la phrase portant sur l'hostie broyée. Ce chapitre ne semblait donc pas poser problème aux lecteurs anglais du XIV et français des $\mathrm{Xv}^{\mathrm{e}}$ et XVI $\mathrm{Xv}^{\mathrm{e}}$ siècles : pour eux, la mention de l'hostie broyée ne réveillait pas le spectre de l'hérésie mais proposait, au contraire, une réflexion intéressante au sujet de ce sacrement.

15 Ainsi le chapitre 15 du Mirouer est-il représentatif de la tension à l'œuvre dans l'ensemble du texte: tendant à l'orthodoxie, il comporte des points d'interprétation délicats, difficiles à résoudre, et qui pouvaient être lus comme mettant en danger le maintien de la communauté chrétienne au XIII ${ }^{\mathrm{e}}$ siècle et au-delà.

\section{Une réception entre parfum de sainteté et odeur de soufre}

Le Mirouer dans son ensemble, et certains de ses chapitres en particulier, ont été lus en 1309 par l'inquisiteur Guillaume de Paris, garant du maintien de la communauté chrétienne, comme la mettant en péril. En conséquence, Marguerite Porete fut jugée hérétique par le tribunal inquisitorial de Paris en 1310 et brûlée en tant que relapse sur la place de Grève le $1^{\text {er }}$ juin de la même année ${ }^{23}$. Marguerite semble avoir accepté son exclusion de la communauté des croyants ${ }^{24}$, mais, lors de son exécution, elle est pleurée par les témoins de sa mort, béguines et laïcs.

Qu'en est-il de la réception du Mirouer après la mort de son auteur? Se place-t-elle sous le signe de la méfiance à l'égard d'un texte remettant en cause la confiance des fidèles envers le clergé ? Ou bien le texte apparaît-il comme tout à fait orthodoxe ?

En observant les milieux dans lesquels ont été copiés les manuscrits du Mirouer des simples ames qui nous sont parvenus, une conclusion s'impose : il s'agit de milieux monastiques, majoritairement masculins, qui n'ont rien d'hétérodoxe. Les manuscrits les plus anciens sont les manuscrits latins : copiés au XIV ${ }^{\mathrm{e}}$ siècle, ils ont appartenu à des couvents italiens. $\mathrm{Au} \mathrm{XIV}^{\mathrm{e}}$ siècle sont aussi copiées les versions italiennes que nous connaissons du Mirouer: l'une de ces copies a été détenue par le monastère franciscain de Capestrano, sis à Venise ou Padoue, et un autre manuscrit est celui d'un particulier. Les manuscrits en médioanglais datent principalement $\mathrm{du} \mathrm{xv}^{\mathrm{e}}$ siècle: ils ont tous appartenu à des couvents chartreux, principalement à Londres. $A u \mathrm{Xv}^{\mathrm{e}}$ siècle, on trouve une version latine chez d'autres Chartreux, à Strasbourg cette fois-ci. Le manuscrit médio-français, du $\mathrm{xv}^{\mathrm{e}}$ siècle, était le bien d'un particulier avant d'être légué au couvent de La Madeleine-lès-Orléans, couvent de religieuses d'inspiration fontevriste. Enfin, au XvI siècle, c'est le couvent bénédictin de Subiaco en Italie qui détient une copie du Mirouer dans un manuscrit préparatoire à une impression, qui ne fut pas réalisée. De tels milieux de copie pourraient sembler garantir une lecture orthodoxe du Mirouer ${ }^{25}$.

19 Si le Mirouer est parfois copié seul, il est, dans d'autres manuscrits, compilé avec d'autres textes. Comment notre traité mystique s'insère-t-il dans ce corpus de textes? À quelles communautés textuelles est-il rattaché? Étrangement, au vu de la condamnation de l'auteur du Mirouer pour hérésie, les textes avec lesquels notre texte a été copié appartiennent à la mystique chrétienne de leur temps. Ainsi est-il copié, au XIV siècle, à côté d'un traité de prières de Richard de Saint-Victor ou de Nicolas de Cuse, d'un traité de piété de saint Bernard, de textes de Richard Rolle de Hampolle sur Margarete Heslyngter 
ou encore entouré de passages d'Augustin et de Jérôme ${ }^{26}$. D'autres manuscrits conservent uniquement le Mirouer ${ }^{27}$ ou l'accompagnent d'un fragment de commentaire ${ }^{28}$. Dans tous les cas, pas de quoi crier à l'anathème ou précipiter un autodafé : les textes qui forment une compilation avec le Mirouer sont des textes spirituels, parfois mystiques. La plupart de leurs auteurs ont écrit à destination de femmes laïques (saint Bernard, Richard de Saint-Victor) ou bien sur des femmes laïques s'étant distinguées par une vie exemplaire sur le plan de la piété (Richard Rolle de Hampole à propos de Margarete Heslyngter). Ces textes se signalent également par l'importance qu'ils accordent à la vie contemplative et à l'ascétisme, voire à l'érémitisme (Richard Rolle de Hampole à propos de Margarete Heslyngter), et par leur inspiration néo-platonicienne (pseudo-Denys, Richard de Saint-Victor, Bernard de Clairvaux, Nicolas de Cuse). Autant de traits doctrinaux communs au Mirouer. Le Mirouer des simples ames a donc été copié dans des milieux monastiques soucieux de contemplation et de mystique, accoutumés à la pensée néo-platonicienne et a été lu dans ces milieux comme parfaitement orthodoxe. Cela souligne le crédit qu'on lui portait. Le risque de persécution dont faisait état le chapitre d'approbation adressé aux lecteurs semble, peut-être temporairement, avoir disparu. Le Mirouer apparaît d'ailleurs toujours dans ces compilations de manière anonyme.

Deux manuscrits dérogent pourtant à la règle. Ils poussent au contraire notre texte du côté de l'hérésie et soulignent la tension entre orthodoxie et hétérodoxie qui le traverse. Le premier est le manuscrit composé en vue du concile de Ferrare portant des propositions extraites du Mirouer : composé en 1438, il fut ensuite détenu par un cardinal ${ }^{29}$. La présence de propositions extraites du Mirouer dans un manuscrit conciliaire ne plaide pas tout à fait en faveur de l'orthodoxie de ce texte : ce concile visait notamment à condamner des hérétiques et s'appuyait pour cela sur les erreurs du Mirouer. Le second est le manuscrit des Chartreux de Strasbourg, dont le texte du Mirouer a été délibérément supprimés ${ }^{30}$. Après le folio où commence le texte, un cahier a en effet été découpé " pour faire disparaître le Miroir ", indique Romana Guarnieri dans son édition ${ }^{31}$. Simple désintérêt ou geste de réaction aux soupçons planant autour du traité ?

Auteur potentiellement hérétique, copies et lectures soucieuses d'orthodoxie : l'histoire du Mirouer illustre la relativité de la définition de l'hérésie. Ce qui semble menacer la communauté chrétienne en un temps donné apparaît, au contraire, comme un instrument de cohésion en un autre. Rappelons que certaines femmes pieuses, comme Marie d'Oignies, furent érigées par l'Église, face à diverses hérésies (ici, le catharisme), comme modèle de piété laïque orthodoxe. Que le Mirouer, œuvre d'une femme jugée relapse, ait pu être attribué à une sainte, Marguerite de Hongrie, dans les manuscrits italiens, n'est qu'un des nombreux paradoxes qui jalonnent l'histoire des hérésies dans l'Occident chrétien au Moyen Âge.

Le Mirouer des simples âmes, texte mystique de la fin du XIII ${ }^{\mathrm{e}}$ siècle, illustre la complexité qui consiste à vouloir demeurer dans une communauté dont on cherche à renouveler l'esprit. Proposant une mystique radicale, élitiste, son auteur porte la singularité de sa parole avec audace jusque devant les garants de son appartenance au groupe, les clercs. Il ne semble pas s'agir de rejeter cette communauté, quoique le texte soit parfois à cet endroit ambigu. Ce dont on peut être sûr, c'est qu'il s'agit de n'être pas rejeté par cette communauté. L'histoire de la réception de ce texte aurait tendance à donner raison à son auteur : copié dans des milieux ecclésiastiques, le Mirouer a été lu à côté des plus grands mystiques du christianisme. Pourtant c'est à une hérétique, Marguerite Porete, qu'il a été attribué au $\mathrm{xx}^{\mathrm{e}}$ siècle. Dès lors, c'est de manière dramatique que la volonté de l'auteur du 
Mirouer aura été mise en échec: rejeté de la communauté dans laquelle son livre souhaitait s'inclure, son auteur en aura péri. L'effacement de son nom aura pourtant permis à son texte de suivre le destin qui avait été conçu pour lui. Cela nous invite à voir dans le procès de Marguerite Porete un cas d'invention d'une hérésie, où l'apparente menace de dissolution de la communauté, concentrée autour d'une personne, se délite dès lors que l'on examine objectivement la complexité de son discours ${ }^{32}$.

\section{NOTES}

1. Monique Zerner, «Hérésies » dans le Dictionnaire du Moyen Âge, dir. Claude Gauvard, Alain de Libera et Michel Zink, Paris, PUF, coll. « Quadrige », 2002, p. 668.

2. Jacques Le Goff, La Civilisation de l'Occident médiéval [1964], Paris, Garnier Flammarion, coll. « Champs Histoire », 2008, p. 289.

3. Les manuscrits latins sont conservés à la Bibliothèque apostolique vaticane, sous les cotes Vat. Lat. 4335 , Rossianus 4, Chigianus B IV 41, Chigianus C IV 85, Vat. Lat. 4 953, et à la Bodleian Library d'Oxford, sous la cote Laud. Lat. 46 ; les manuscrits anglais sont conservés au British Museum de Londres (Add. Ms. 37 790), aux bibliothèques de St John's College (C 21) et de Pembroke à Cambridge, sous la cote Ms Add. 221 ; les manuscrits italiens se trouvent dans les bibliothèques nationales de Florence (Riccardiano 1468 ) et de Naples (XII F 5), ou encore à celles de Vienne (Palatino 15 093) et de Budapest, sous la cote Octoboniano italiano 15. Quant au manuscrit en moyen français, il est conservé au musée Condé de Chantilly sous la cote F XIV 26 (ancien 986), catalogue 157. Plus récemment, des citations du Mirouer en langue picardisante ont été

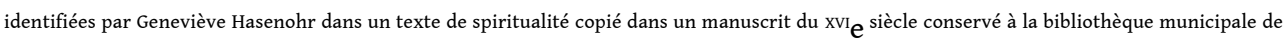
Valenciennes sous la cote 0239 .

4. Par «Église dans l'Église", nous désignons la communauté d'âmes élues regroupées dans le Mirouer sous le terme de «Saincte Eglise la Grande ».

5. Le pronom objet «les» en début de phrase renvoie aux destinataires du livre, auxquels renvoyait déjà le pronom relatif «qui » dans la proposition relative « pour qui je ne sais ni ne veux le savoir » de la première phrase. Le Miroir se caractérise par un emploi des pronoms dont les référents peuvent être très difficiles à identifier. Voici le texte original latin: «Ego creatura a creante condita, mediante qua Creator de se fecit hunc librum, pro quibus nescio nec scire uolo, quia hoc non debeo uelle. Sufficit enim mihi si est in secreto scire diuinae sapientiae et in spe. Saluto autem eos per amorem pacis caritatis in altissima Trinitate, quae eos dirigere dignetur, dicendo in eis testimonium uitae eorum per recordationem clericorum qui audierunt / istum librum » (Speculum simplicium animarum, éd. Paul Verdeyen, Turnhout, Brepols, 1991, c. 140, p. 405, 1. 1-9).

6. Les ouvrages se rattachant au genre du « miroir » portent souvent un titre indiquant ce genre et le groupe auquel il se destine. C'est le cas par exemple du Miroir des Vierges (Speculum virginium) de Vincent de Beauvais.

7. «Les clercs qui ont entendu ce livre ». Qui furent ces ecclésiastiques ? La suite du chapitre nous l'apprend. Le premier clerc, un franciscain, avoue qu'il ne comprend pas ce livre, trop «haut» (altus) pour lui et recommande de le diffuser avec parcimonie. Le deuxième clerc, un cistercien, s'appuie sur l'Écriture (per Scripturas) pour garantir pour vrai tout ce que ce livre dit. Le dernier clerc, un maître de théologie de l'université de Paris originaire de Liège, Godefroid de Fontaine, recommande également à l'auteur du Mirouer de ne pas diffuser largement ce livre, qui risque de convaincre certains lecteurs de suivre une vie qui ne leur est pas destinée. Il reconnaît toutefois la force et la ferveur de l'esprit de son auteur, comme son caractère exceptionnel, et ajoute que l'exercice (usum) spirituel décrit par ce livre est divin et non humain et qu'il est le seul à permettre de parvenir à la vie divine.

8. L'édition du texte en moyen français et sa traduction en italien se trouvent dans Margherita Porete, Lo Specchio delle anime semplici, éd. Romana Guarnieri, Roma, San Paolo, 1994, p. 122-126. 
9. Ibid., ch. $121,1.24$, p. 438.

10. Monique Zerner définit l'hérésie comme le «refus de l'autorité de l'Église » dans l'article « Hérésie » du Dictionnaire du Moyen Âge, art. cit., p. 668.

11. Georges Duby, Le Chevalier, la femme et le prêtre, Paris, Hachette, coll. «Pluriel », 1981, p. 118. Voir aussi Didier Lett, Hommes et femmes au Moyen Âge. Histoire du genre, $\mathrm{XII}^{\mathrm{e}}-\mathrm{XV}^{\mathrm{e}}$ siècle, Paris, Armand Colin, coll. « Cursus histoire », 2013.

12. Le terme de «communion » au sens de participation au sacrement de l'Eucharistie n'est pas encore très répandu au XIII ${ }^{\mathrm{e}}$ siècle. On parle d'eucharistia, c'est-à-dire, étymologiquement, d'« action de grâce » (eukharistia en grec), de Corpus christi, de Saint Sacrement et de Sacrement de l'autel, mais peu de communio. Le mot n'apparaît pas dans le canon 21 de Latran IV (1215). Pourtant, c'est bien le lien du fidèle à son Église qui est en jeu : à défaut de cette participation pascale au sacrement eucharistique et de sa confession annuelle, ce dernier sera exclu de la communauté catholique. Ainsi, quoique le mot communio ne se soit pas encore imposé au XIII siècle, le sème d'intégration à une communauté est bien présent dans le terme d'Eucharistie tel qu'employé par les textes conciliaires.

13. Après s'être adressé à ses auditeurs et avoir présenté le but de son ouvrage en dressant un parallèle avec l'épisode courtois d'un roman de chevalerie populaire, le Roman d'Alexandre (chapitres 1 et 2), l'auteur explique la voie de purification menant à Dieu de trois manières : d'abord en dialogue exclusif avec Raison (chapitres 3 et 9), puis à l'attention des actifs (chapitres 10 à 12) et enfin à l'attention des contemplatifs (chapitre 13) sous forme d'un mini-traité en neuf points comme il en fleurissait dans la littérature spirituelle, et notamment béguinale, de l'époque .

14. Ainsi, ce sont deux femmes pieuses, sainte Julienne de Cornillon et la bienheureuse Ève de Liège, qui sont à l'origine de l'institution de la Fête du Corps du Christ, dite aussi Fête du Saint-Sacrement. Cette fête se tient soixante jours après Pâques. Elle offre l'occasion d'une deuxième communion, la première, et la seule exigée par l'Église, étant annuelle et pascale. L'excès de cette dévotion, que l'on peut rapprocher de la popularité de la mystique nuptiale suite aux sermons cisterciens, fut critiqué par des sectes hérétiques comme les cathares dans le sud de la France, les lollards en Angleterre ou les hussites dans le Saint Empire germanique.

15. Jacques Le Goff, La Civilisation de l'Occident médiéval, op. cit., p. 292.

16. « Il n'y a qu'une seule Église universelle des fidèles, [...] dans laquelle Jésus-Christ est le prêtre et la victime, dont le corps et le sang sont véritablement dans le sacrement de l'autel sous les espèces du pain et du vin; le pain étant transsubstantié au corps de Jésus-Christ, et le vin en son sang, par la puissance divine; afin que, pour rendre le mystère de l'unité parfait, nous recevions du sien ce qu'il a reçu du nôtre » : Latran IV (1215), canons 1 et 21.

17. Margherita Porete, Lo Specchio delle anime semplici, op. cit., ch. 15, p. 180.

18. Ibid.

19. Cette base de données est accessible à l'adresse suivante : http://quodlibase.ehess.fr/.

20. Pierre de Jean Olivi, Quodlibeta quinque, éd. Stefano Defraia, Grottaferrata, Frati editori di Quaracchi, 2002. Cette mention d'une hostie broyée peut avoir été lue comme faisant référence à des pratiques déviantes associées à l'hostie. En effet, les procès pour sorcellerie pour lesquels nous disposons de traces, quoique de beaucoup postérieures à la rédaction du Mirouer, n'en sont pas moins instructifs quant à l'usage de l'hostie qui y est reproché, à tort ou à raison, aux accusées : elle se trouve au cœur des messes noires et autres sabbats. On trouve des procès pour sorcellerie à Valenciennes dès 1350 : Catherine Pavot, «Procès précoces de sorcellerie dans la prévôté-le-comte de Valenciennes (1350-1378) », Revue du Nord, vol. 362, n 4, 2005, p. 753-761 et Frédéric Delacroix, Les Procès de sorcellerie au XVII ${ }^{e}$ siècle, Paris, La Nouvelle Revue, 1894, p. 96-97.

21. Voir Jean-Louis Schefer, L'Hostie profanée. Histoire d'une fiction théologique, Paris, POL., 2007.

22. Jan Huizinga, Le Déclin du Moyen Âge, Paris, Payot, coll. «Petite bibliothèque », 1967, p. 160. Sur la prédication aux béguines, voir les travaux de Nicole Bériou, notamment La Prédication de Ranulphe de la Houblonnière. Sermons aux clercs et aux simples gens à Paris au XIII siècle, 2 vol., Paris, Institut d'Études augustiniennes, 1987 et L'Avènement des maîtres de la parole : la prédication à Paris au XIII siècle, 2 vol., Paris, Institut d'Études Augustiniennes, 1998. 
23. Guillaume de Nangis, Chronique du règne de Philippe le Bel (1285-1314), trad. François Guizot, Paris, 1843, éd. Yves Germain et Éric de Bussac, Clermont-Ferrand, Paléo, coll. « Histoire. Accès direct ", 2011, p. 128.

24. Ibid. Elle ne cherche pas à se défendre pendant son procès et donne des signes de piété avant son exécution.

25. Il faut pourtant se souvenir que ce sont souvent les bibliothèques des couvents qui ont le mieux conservé les manuscrits grâce auxquels nous avons aujourd'hui accès aux textes. Nombreux sont les manuscrits détenus par des particuliers qui ont disparu. Il est donc difficile d'aboutir à quelque conclusion que ce soit concernant le type de lecture du Mirouer à partir du milieu dans lequel ont été copiés les manuscrits qui nous en sont parvenus.

26. Respectivement: Rossianus 4, Chigianus C IV 85, Add. Ms 37790 et Laud. Lat. 46.

27. Manuscrit de Chantilly.

28. D'une phrase du pseudo-Denys pour Rossianus 4.

29. Le cardinal Sirleto (1512-1585).

30. Manuscrit possédé par les Chartreux de Strasbourg, $\mathrm{f}_{\mathrm{O}^{70 \mathrm{v}} \mathrm{O}}$.

31. Marguerite Porete, Le Mirouer des simples ames, éd. Romana Guarnieri, Turnhout, Brepols, coll. «Corpus christianorum - Continuatio Mediaeualis », 1986.

32. Nous empruntons l'idée d'invention de l'hérésie à l'ouvrage collectif dirigé par Monique Zerner, Inventer l'hérésie? Discours polémiques et pouvoirs avant l'Inquisition, Paris, CNRS, c oll. «Études médiévales " $\mathrm{n}^{\circ} 2$, 1998. Enfin, au sujet des hérésies, voir le volume dernièrement paru, dirigé par André Vauchez, Les Hérétiques au Moyen Âge. Suppôts de Satan ou chrétiens dissidents?, Paris, CNRS, 2014.

INDEX

Mots-clés : orthodoxie, hérésie

Keywords : orthodoxy, heresy

\section{AUTEUR}

\section{ELODIE PINEL}

Université Paris Ouest Nanterre La Défense 